\title{
Comparative Analysis of the Effects of Olive Oil Hydroxytyrosol and Its 5-S-Lipoyl Conjugate in Protecting Human Erythrocytes from Mercury Toxicity
}

\author{
Arbace Officioso, ${ }^{1}$ Lucia Panzella $\left(\mathbb{D},{ }^{2}\right.$ Fabiana Tortora, ${ }^{1}$ Maria Laura Alfieri, ${ }^{2}$ \\ Alessandra Napolitano $\left(\mathbb{D},{ }^{2}\right.$ and Caterina Manna $\mathbb{1}^{1}$ \\ ${ }^{1}$ Department of Biochemistry, Biophysics and General Pathology, University of Campania "Luigi Vanvitelli", Naples, Italy \\ ${ }^{2}$ Department of Chemical Sciences, University of Naples "Federico II", Naples, Italy
}

Correspondence should be addressed to Alessandra Napolitano; alesnapo@unina.it and Caterina Manna; caterina.manna@unicampania.it

Received 20 September 2017; Accepted 4 March 2018; Published 12 April 2018

Academic Editor: Glenda Gobe

Copyright () 2018 Arbace Officioso et al. This is an open access article distributed under the Creative Commons Attribution License, which permits unrestricted use, distribution, and reproduction in any medium, provided the original work is properly cited.

Oxidative stress is one of the underlying mechanisms of the toxic effects exerted by mercury (Hg) on human health. Several antioxidant compounds, including the olive oil phenol hydroxytyrosol (HT), were investigated for their protective action. Recently, we have reported that 5-S-lipoylhydroxytyrosol (Lipo-HT) has shown increased antioxidant activities compared to HT and exerted potent protective effects against reactive oxygen species (ROS) generation and oxidative damage in human hepatocellular carcinoma HepG2 cell lines. In this study, the effects of Lipo-HT and HT on oxidative alterations of human erythrocytes induced by exposure to $40 \mu \mathrm{M} \mathrm{HgCl}_{2}$ were comparatively evaluated. When administered to the cells, Lipo-HT $(5-20 \mu \mathrm{M})$ proved nontoxic and it decreased the Hg-induced generation of ROS, the hemolysis, and the depletion of intracellular GSH levels. At all tested concentrations, Lipo-HT exhibited higher ability to counteract Hg-induced cytotoxicity compared to HT. Model studies indicated the formation of a mercury complex at the SH group of Lipo-HT followed by a redox reaction that would spare intracellular GSH. Thus, the enhanced erythrocyte protective action of Lipo-HT from Hg-induced damage with respect to HT is likely due to an effective chelating and reducing ability toward mercury ions. These findings encourage the use of Lipo-HT in nutraceutical strategies to contrast heavy metal toxicity in humans.

\section{Introduction}

Hydroxytyrosol (HT) is a dietary phenolic antioxidant compound, naturally present in virgin olive oil, contributing to its great oxidative stability and prolonged shelf life [1]. HT is endowed with several pharmacological properties [2-4], including anti-inflammatory [5], antiatherogenic [6], and anticancer activities [7]. Mechanisms underlying HT biological effects include both radical scavenging properties and metal chelating activity [8-11]. Cytoprotective effects of HT against xenobiotic compounds have been reported, including protection from acrolein-induced DNA damage [12], acrylamide-induced mitochondrial dysfunction [13], and carbon tetrachloride-induced oxidative stress [14].

Mercury $(\mathrm{Hg})$ is a highly toxic, redox-active heavy metal which represents one of the main agents responsible for environmental pollution $[15,16]$. The health consequences of human exposure to $\mathrm{Hg}$ can be severe $[17,18]$ and include renal injury [19] and neuronal disorders [20]. In this respect, $\mathrm{Hg}$ is considered a potential contributing factor to Alzheimer's and Parkinson's diseases [21]. Red blood cells (RBC) may also represent an important target of $\mathrm{Hg}$ toxicity. This metal, indeed, preferentially accumulates in these cells and induces morphological changes which increase their procoagulant activity [22-24]. Finally, an 
increasing body of data suggests a positive correlation between $\mathrm{Hg}$ exposure and the onset of cardiovascular diseases [25].

The increased formation of reactive oxygen species (ROS) is generally agreed to be one of the key mechanisms responsible for $\mathrm{Hg}$-induced toxicity [26-28]. Mercury is endowed with high affinity for sulfhydryl groups, and it is therefore able to react with low-molecular-weight thiols, including glutathione (GSH) $[29,30]$.

Indeed, the cellular levels of GSH have been shown to significantly decrease following $\mathrm{Hg}$ exposure, with consequent impairment of the antioxidant defence system [31]. Following the oxidative stress hypothesis of $\mathrm{Hg}$ toxicity, a number of antioxidants [32, 33], including phenolic compounds [34-36], have been proposed and tested for their protective action. In this connection, we recently provided experimental evidence that $\mathrm{HT}$ has the potential to modulate cytotoxicity and the oxidative stress induced in human RBC by Hg treatment $[37,38]$. Using the scanning electron microscopy technique, we also showed that HT treatment significantly reduces echinocyte formation [37], a morphological $\mathrm{RBC}$ alteration reported to correlate with increased coagulability of these cells [39]. Furthermore, a recent study by Mohan et al. highlighted the efficacy of HT in preventing methylmercury-induced genotoxicity and apoptosis in IMR-32 human neuroblastoma cells. HT was shown to inhibit methylmercury-induced neuronal cell dysfunction as highlighted by the decrease in ROS formation and maintenance of an efficient endogenous defence system, including GSH levels and superoxide dismutase and catalase activities [40].

In the last few years, several HT derivatives with enhanced antioxidant and pharmacological properties have been described [41-46]. Among these, 5-S-lipoylhydroxytyrosol (Lipo-HT), synthesized by conjugation of HT with the biologically relevant thiol dihydrolipoic acid, showed increased antioxidant activities compared to HT in several chemical assays and exerted potent protective effects against ROS generation and oxidative cell damage in human hepatocellular carcinoma HepG2 cell lines (Figure 1) [47, 48]. Insertion of the sulfur-containing chain was found to exert a crucial influence on the reactivity of the adjacent catechol system conferring a more pronounced lipophilic character on the core system and resulting in an overall potentiation of the antioxidant activity of HT. This effect was also demonstrated in the case of other naturally occurring catechols $[49,50]$. Because of the peculiar combination in its molecular scaffold of a number of redox-active groups, Lipo-HT exerts a multidefence antioxidant action through different mechanisms, including $\mathrm{H}$-atom release, ferric and cupric ion reduction, and $\mathrm{OH}$ radical scavenging [48].

The aim of this study was to test the ability of Lipo-HT to prevent human $\mathrm{RBC}$ from the oxidative alterations induced by $\mathrm{Hg}$ treatment in comparison with the parent HT. These cells are a unique cellular model for in vitro studies which investigate oxidative stress-related alterations as well as $\mathrm{Hg}$ toxicity $[23,24,37,38]$. Mercury(II)chloride $\left(\mathrm{HgCl}_{2}\right)$ was selected for running the experiments since mercury is a well-known oxidative stress inducer [51].<smiles>OCCc1ccc(O)c(O)c1</smiles><smiles>O=C(O)CCCCC(S)CCSc1cc(CCO)cc(O)c1O</smiles><smiles>O=C(O)CCCCC1CCSS1</smiles>

Lipoic acid

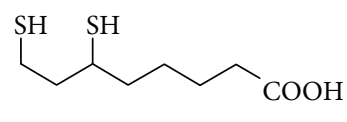

Dihydrolipoic acid
FIGURE 1

The rationale of testing Lipo-HT in this toxicity model system stems from the possibility of exploiting, in addition to the antioxidant power of the catechol moiety activated by the adjacent thioalkyl group, the free $\mathrm{SH}$ group of dihydrolipoic acids capable of effectively chelating $\mathrm{Hg}$. Cytoprotective effects were indeed observed in the case of this conjugate, whose mechanisms were initially investigated at the molecular level by model experiments.

\section{Materials and Methods}

2.1. Chemicals. $2^{\prime}, 7^{\prime}$-Dichlorodihydrofluorescein diacetate (DCFH-DA), mercuric chloride $\left(\mathrm{HgCl}_{2}\right)$, tyrosol, lipoic acid, 5,5'-dithiobis(2-nitrobenzoic acid) (DTNB) (Ellman's reagent), and HT were from Sigma Chemical Co. All other chemicals used were of the highest purity grade available.

2.2. Methods. UV/Vis spectra were recorded on a Jasco V-730 spectrophotometer. HPLC analysis was carried out on an Agilent instrument equipped with a UV detector set at $254 \mathrm{~nm}$. The chromatographic separation was achieved on a Phenomenex SphereClone ODS column $(250 \mathrm{~mm} \times 4.6 \mathrm{~mm}$, $5 \mu \mathrm{m})$ using binary gradient elution conditions as follows: $0.1 \%$ trifluoroacetic acid (solvent $\mathrm{A}$ ) and acetonitrile (solvent B), from $5 \%$ to $90 \% \mathrm{~B}, 0-45 \mathrm{~min}$, and flow rate $0.7 \mathrm{~mL} / \mathrm{min}$. LC/MS analyses were run on a LC/MS ESI-TOF 1260/ 6230DA Agilent instrument operating in positive ionization mode in the following conditions: nebulizer pressure 35 psig; drying gas (nitrogen) $8 \mathrm{~L} / \mathrm{min}, 325^{\circ} \mathrm{C}$; capillary voltage $3500 \mathrm{~V}$; and fragmentor voltage $175 \mathrm{~V}$. An Eclipse Plus C18 column, $150 \times 4.6 \mathrm{~mm}, 5 \mu \mathrm{m}$, at a flow rate of $0.4 \mathrm{~mL} / \mathrm{min}$ was used, with the same mobile phase as above.

2.3. Synthesis of 5-S-Lipoylhydroxytyrosol (Lipo-HT). The procedure previously described was adopted [48]. The reaction was carried out on tyrosol $(1.0 \mathrm{~g})$ affording Lipo-HT in about $30 \%$ yield. The purity of the compound was evaluated (>95\%) by HPLC and ${ }^{1} \mathrm{H}$ NMR analysis.

2.4. Preparation of $R B C$ and Treatment with $\mathrm{Hg}$. The RBC fraction was obtained from whole blood obtained from human healthy volunteers deprived of leucocytes and platelets by filtration on a nylon net, washed twice with isotonic saline solution $(0.9 \% \mathrm{NaCl})$, and finally resuspended with buffer A ( $5 \mathrm{mM}$ Tris- $\mathrm{HCl}$ containing $0.9 \%$ 
$\mathrm{NaCl}, 1 \mathrm{mM} \mathrm{MgCl}$, and $2.8 \mathrm{mM}$ glucose, $\mathrm{pH} 7.4$ ) to obtain a $10 \%$ hematocrit. Intact $\mathrm{RBC}$ were incubated at $37^{\circ} \mathrm{C}$ with $40 \mu \mathrm{M} \mathrm{HgCl} 2$ for $4 \mathrm{~h}$. For the experiments with Lipo-HT or HT, stock solutions $(100 \mathrm{mM})$ were prepared in DMSO. Just before the experiments, these solutions were diluted to $1 \mathrm{mM}$ with the isotonic saline solution and added to the incubation medium to obtain the desired concentration 5 min before addition of $\mathrm{Hg}$. As a control, the effects of the highest volume of DMSO used on RBC in the presence of $\mathrm{Hg}$ were also evaluated and found to be negligible. $\mathrm{RBC}$ from each donor were used for a single assay in triplicate. Each experiment was repeated on RBC obtained from three different donors.

2.5. Determination of Hemolysis. The extent of hemolysis was determined spectrophotometrically, according to Tagliafierro et al. [37]. At the end of the incubation, the reaction mixture was centrifuged at $1100 \mathrm{~g}$ for $5 \mathrm{~min}$ and the hemoglobin $(\mathrm{Hb})$ released was evaluated by measuring the absorption of the supernatant at $540 \mathrm{~nm}$. Packed RBC were hemolyzed with ice-cold distilled water at $40: 1 \mathrm{v} / \mathrm{v}$, the lysate was centrifuged at $1500 \mathrm{~g}$ for 10 minutes, and the supernatant (B) was absorbed at $540 \mathrm{~nm}$. The percentage of hemolysis was calculated as the ratio of the readings $(\mathrm{A} / \mathrm{B}) \times 100$.

2.6. Determination of ROS. To quantify ROS generation, the dichlorofluorescein (DCF) assay was used according to Tagliafierro et al. [37]. Intact RBC were incubated with DCFH-DA at $10 \mu \mathrm{M}$ final concentration for $15 \mathrm{~min}$ at $37^{\circ} \mathrm{C}$. After centrifugation at $1200 \mathrm{~g}$ for $5 \mathrm{~min}$, the supernatant was removed and the hematocrit value was adjusted to $10 \%$ with buffer $\mathrm{A}$. RBC were then treated with $\mathrm{HgCl}_{2}$ in the dark. At the end of incubation, $20 \mu \mathrm{L}$ of RBC were diluted in $2 \mathrm{~mL}$ of water and the fluorescence intensity of the oxidized derivative DCF was recorded $\left(\lambda_{\text {ex }} 502 \mathrm{~nm} ; \lambda_{\text {em }} 520 \mathrm{~nm}\right)$. The results were expressed as fluorescent intensity/mg of $\mathrm{Hb}$.

2.7. Quantification of Intracellular GSH. The intracellular GSH content was determined spectrophotometrically by reaction with DTNB reagent, according to van den Berg et al. [52]. The samples $(0.25 \mathrm{~mL})$ described above were centrifuged, the supernatants were removed, and RBC were lysed by the addition of $0.6 \mathrm{~mL}$ of ice-cold water. Proteins were precipitated by the addition of $0.6 \mathrm{~mL}$ ice-cold metaphosphoric acid solution $(1.67 \mathrm{~g}$ metaphosphoric acid, $0.2 \mathrm{~g}$ EDTA, and $30 \mathrm{~g} \mathrm{NaCl}$ in $100 \mathrm{~mL}$ of water). After incubation at $4^{\circ} \mathrm{C}$ for $5 \mathrm{~min}$, the protein precipitate was removed by centrifugation at $18000 \mathrm{~g}$ for $10 \mathrm{~min}$ and $0.45 \mathrm{~mL}$ of the supernatant was mixed with an equal volume of $0.3 \mathrm{M} \mathrm{Na}_{2} \mathrm{HPO}_{4} .100 \mu \mathrm{L}$ of DTNB solution (20 mg DTNB plus $1 \%$ of sodium citrate in $100 \mathrm{~mL}$ of water) was then added to the sample, and after a 10 min incubation at room temperature, the absorbance of the sample was read against the blank at $412 \mathrm{~nm}$.

2.8. Reaction of Lipo-HT or HT with $\mathrm{Hg}^{2+}$ Ions. $5 \mu \mathrm{L}$ of a $100 \mathrm{mM} \mathrm{HgCl}{ }_{2}$ solution was added to $5 \mathrm{~mL}$ of buffer (pH 7.4), followed by $5 \mu \mathrm{L}$ of a $50 \mathrm{mM}$ DMSO solution of Lipo-HT or HT $(100 \mu \mathrm{M}$ and $50 \mu \mathrm{M}$ final concentrations for $\mathrm{Hg}^{2+}$ and Lipo-HT or HT, resp.). The reaction mixture was taken under stirring and periodically analyzed by HPLC, LC/MS, and UV/Vis spectroscopy. When required, the reaction mixture was taken to $\mathrm{pH} 3$ with $4 \mathrm{M} \mathrm{HCl}$ before analysis. In other experiments, aliquots $(1.5 \mathrm{~mL})$ of the reaction mixture of Lipo-HT were periodically withdrawn, added with $11 \mu \mathrm{L}$ of a $10 \mathrm{mM}$ Ellman's reagent solution in $0.1 \mathrm{M}$ phosphate buffer ( $\mathrm{pH} 7.4$ ), and after $15 \mathrm{~min}$ the absorbance at $412 \mathrm{~nm}$ was read. In other experiments, the reaction was run: (i) in the absence of $\mathrm{HgCl}_{2}$ and (ii) under an argon atmosphere. Methanooxocinobenzodioxinone derivatives were obtained by tyrosinase-catalyzed oxidation of HT as previously described [53].

2.9. Statistical Analysis. Data are shown as means \pm SE. The significance of differences was determined by one-way ANOVA followed by a post hoc Dunnett multiple comparison test with significance set at $p<0.05$. GraphPad Prism 5 was used for statistical analysis.

\section{Results and Discussion}

3.1. Cytoprotective Effects of Lipo-HT and HT on Hg-Induced Oxidative Alterations in RBC. The ability of natural and biobased phenolic antioxidants to counteract $\mathrm{Hg}$-induced cytotoxicity was investigated using olive oil HT and its conjugate with dihydrolipoic acid, Lipo-HT, which in in vitro tests proved highly efficient in counteracting the action of ROS and associated cellular damage [48]. Lipo-HT was prepared from tyrosol and dihydrolipoic acid according to a procedure previously developed [48].

Intact $\mathrm{RBC}$ were exposed in vitro to $40 \mu \mathrm{M} \mathrm{HgCl}_{2}$. Based on our previous results [37] on the dose-dependency of $\mathrm{Hg}$ toxic effects in RBC, the concentration of $40 \mu \mathrm{M}$ was taken as the optimal dose to study the oxidative stress-mediated cytotoxicity in our in vitro model. Moreover, it is worth noting that comparable concentrations have been found in the blood of individuals exposed to peculiar working environments like gold mines [16]. Cellular lysis, ROS formation, and intracellular GSH levels were evaluated in RBC following $4 \mathrm{~h}$ incubation.

Prolonged $\mathrm{Hg}$ treatment is associated with severe hemolysis; therefore, cell lysis was evaluated by measuring hemoglobin release in the medium upon cell exposure to $\mathrm{HgCl}_{2}$. Data in Figure 2 show a dramatic increase in the hemolytic process confirming the cytotoxicity resulting from exposure of cells to $\mathrm{HgCl}_{2}$. Both compound LipoHT and HT are effective in preventing the toxic effect of the heavy metal as highlighted by the decrease in hemolysis. At all tested concentrations, Lipo-HT shows an enhanced ability over HT on Hg-induced cytotoxicity. Comparable protective effects are provided by Lipo-HT at concentrations halved with respect to those of HT in a statistically significant mode.

Figure 3 reports ROS production in the presence of $\mathrm{Hg}^{2+}$ and varying amounts of Lipo-HT or HT in the concentration range $5-20 \mu \mathrm{M}$ as determined by DCF fluorescence assay. The Hg-induced ROS generation is dose-dependently prevented in the presence of increasing concentrations of either Lipo-HT or HT, a significant effect being observable starting 


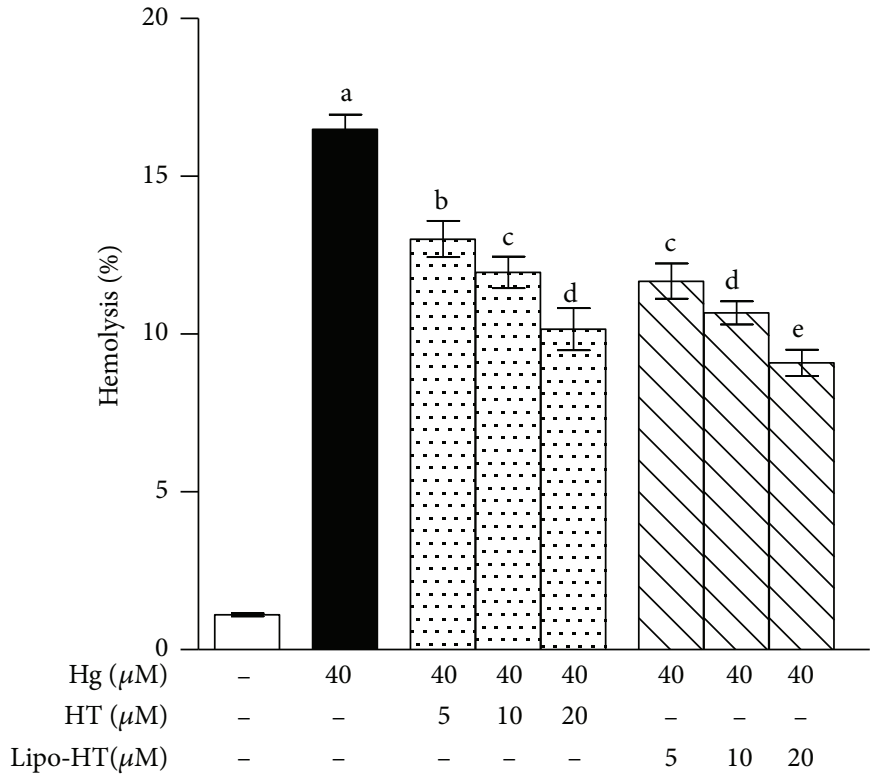

FIGURE 2: Effect of HT and Lipo-HT on Hg-induced hemolysis. Cells were treated with $\mathrm{HgCl}_{2}$ at $40 \mu \mathrm{M}$ for $24 \mathrm{~h}$ in the presence of increasing concentrations of the selected compounds. Data are the means \pm SE $(n=9)$. Statistical analysis was performed with one-way ANOVA followed by Dunnett's test $(p<0.05)$. Means with different letters are significantly different.

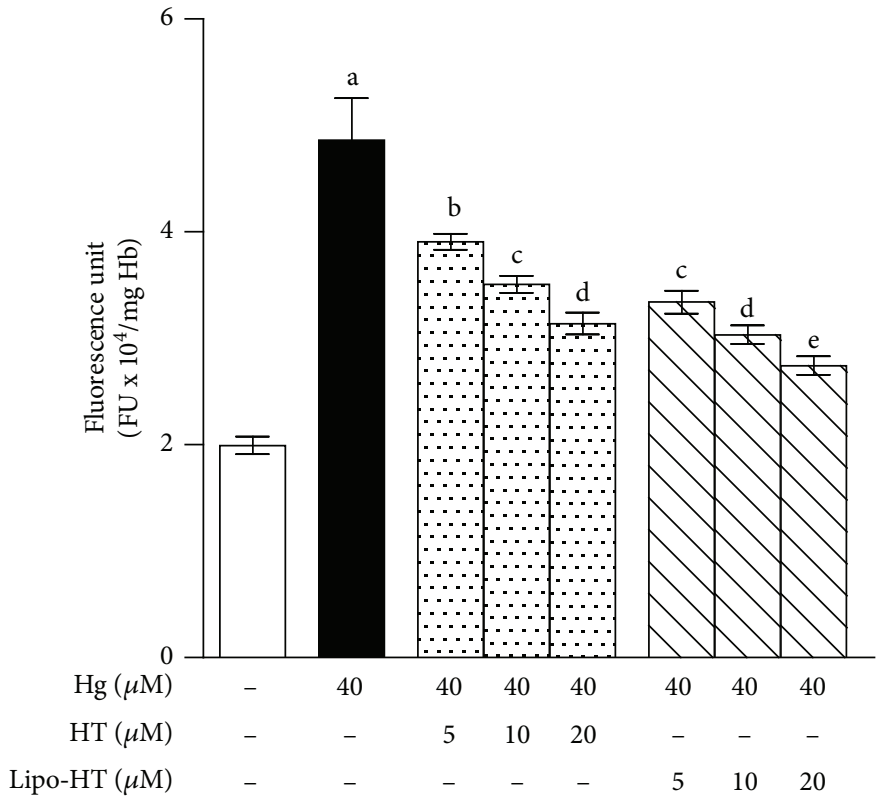

Figure 3: Effect of HT and Lipo-HT on Hg-induced ROS production in RBC. Cells were treated with $\mathrm{HgCl}_{2}$ at $40 \mu \mathrm{M}$ for $4 \mathrm{~h}$ in the presence of increasing concentrations of the selected compounds. ROS production was evaluated by means of the fluorescent probe DCF. Data are the means \pm SE $(n=9)$. Statistical analysis was performed with one-way ANOVA followed by Dunnett's test $(p<0.05)$. Means with different letters are significantly different.

from a concentration as low as $5 \mu \mathrm{M}$. Interestingly, Lipo-HT appears more effective than HT, producing a $57 \%$ decrease of ROS production with respect to the basal level at $5 \mu \mathrm{M}$ versus an only $34 \%$ decrease for HT at the same concentration.

To confirm the observed markedly protective action of Lipo-HT on the specific events that ultimately result in $\mathrm{Hg}$ toxicity, the effect of the compounds under study on GSH intracellular concentrations was evaluated. $\mathrm{Hg}^{2+}$ specifically binds to biological thiols, including GSH, and is able to induce their oxidation with consequent depletion of the intracellular levels. This is generally regarded as a most critical corollary of $\mathrm{Hg}$ toxicity, resulting in the impairment of the antioxidant defence system. Data in Figure 4 refer to the levels of GSH in RBC as determined spectrophotometrically by Ellman's assay. Following cell exposure to $\mathrm{Hg}^{2+}$, the GSH level is significantly reduced with respect to the control 


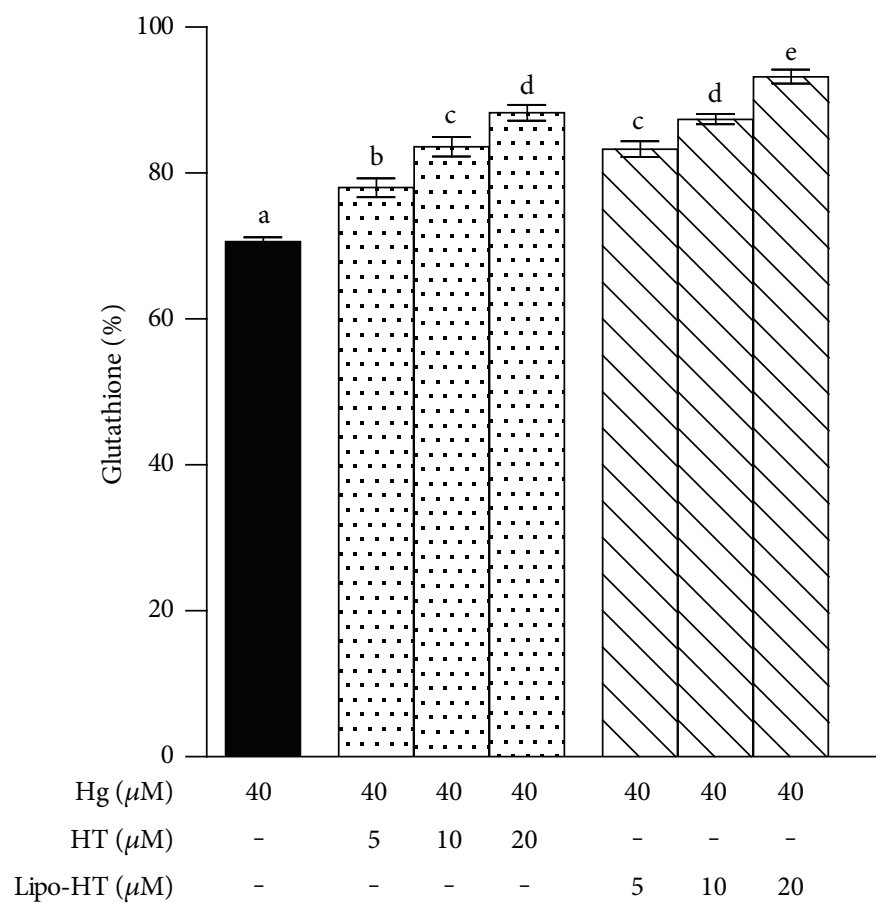

Figure 4: Effect of HT and Lipo-HT on Hg-induced GSH decrease in RBC. Cells were treated with $40 \mu \mathrm{M} \mathrm{HgCl}_{2}$ for $4 \mathrm{~h}$ in the presence of increasing concentrations of the selected compounds. Data are the means \pm SE $(n=9)$. Statistical analysis was performed with one-way ANOVA followed by Dunnett's test $(p<0.05)$. Means with different letters are significantly different.

RBC. Also in this case, coincubation with Lipo-HT at $5 \mu \mathrm{M}$ prevents GSH depletion by about $30 \%$, an effect that is obtained with $10 \mu \mathrm{M}$ HT.

To assess the role of the lipoyl side chain in the observed protective action of Lipo-HT, in other experiments the effects of lipoic acid on Hg-induced toxicity in $\mathrm{RBC}$ were investigated. It is clear that this compound could not fully model dihydrolipoic acid that, however, was not possible to include in the controls due to its high instability. Lipoic acid proved to be quite efficient in controlling the $\mathrm{Hg}$-induced increase of ROS levels with effects at $20 \mu \mathrm{M}$ statistically comparable to those of $\mathrm{HT}$ at the same concentration and those exerted by Lipo-HT at $10 \mu \mathrm{M}$. On the other hand, at 5 and $10 \mu \mathrm{M}$, lipoic acid did not restore the levels of GSH compared to the control in a statistically significant manner and only at the highest dose tested $(20 \mu \mathrm{M})$ did it produce effects statistically comparable to those obtained with $\mathrm{HT}$ at $5 \mu \mathrm{M}$.

3.2. Analysis of the Reaction of Lipo-HT and HT with $\mathrm{Hg}^{2+}$ Ions at pH7.4. In order to obtain information about the mechanisms responsible for the protective effect of Lipo$\mathrm{HT}$ against the damage induced to RBC by $\mathrm{Hg}$ ions, in other experiments the course of the reaction of Lipo-HT $(50 \mu \mathrm{M})$ with $\mathrm{Hg}^{2+}(100 \mu \mathrm{M})$ at $\mathrm{pH} 7.4$ was followed by HPLC and LC/MS. An almost complete consumption of Lipo-HT was observed after $5 \mathrm{~min}$, with concomitant formation of two major products eluted at about 22 and 25 min (Figure 5(a)). This latter showed pseudomolecular ion peaks $[\mathrm{M}+\mathrm{H}]^{+},[\mathrm{M}$ $+\mathrm{Na}]^{+}$, and $[\mathrm{M}+\mathrm{K}]^{+}$at $m / z 921,943$, and 959 , in that order, suggestive of a 2:1 complex of Lipo-HT with Hg. Consistently with the presence of the seven stable isotopes of $\mathrm{Hg}$ (with ${ }^{202} \mathrm{Hg}$ being the most abundant at 29.86\%), these peaks showed a distinct isotopic pattern (Figure 5(b)), which provided further evidence for the formation of the complex [54]. Ellman's assay [55] indicated a more than 90\% abatement of sulfhydryl groups in the reaction mixture after $5 \mathrm{~min}$, pointing to the involvement of the thiolate moiety rather than the catechol unit in $\mathrm{Hg}$-complex formation.

The MS spectrum of the compound eluted at $22 \mathrm{~min}$ was characterized by pseudomolecular ion peaks $[\mathrm{M}+\mathrm{Na}]^{+}$and $[\mathrm{M}+\mathrm{K}]^{+}$at $m / z 381$ and 397 , respectively. A $\left[\mathrm{M}-\mathrm{H}_{2} \mathrm{O}+\mathrm{H}\right]^{+}$ at $\mathrm{m} / z 341$ together with $[2 \mathrm{M}+\mathrm{Na}]^{+}$and $[3 \mathrm{M}+\mathrm{K}]^{+}$at $\mathrm{m} / z$ 739 and 1113, respectively, were also present (Figure 5(c)). These data were suggestive of an oxidation product of Lipo-HT, likely a thioketone as shown in Figure 6. HPLC and LC/MS analysis of the mixture at $1 \mathrm{~h}$ reaction time revealed that the Lipo-HT/Hg complex had disappeared, and it also revealed the presence of thioketone as the only residual product.

In control experiments run in the absence of $\mathrm{Hg}$, only a $20 \%$ consumption of Lipo-HT was observed after $1 \mathrm{~h}$, and no other product could be detected, apart from traces of thioketone and of the disulfide of Lipo-HT [48]. Moreover, a complete consumption of Lipo-HT, together with the formation of the products eluted at 22 and $25 \mathrm{~min}$, was observed even when the reaction was run under an argon atmosphere, ruling out a possible role of oxygen in the oxidation reaction.

Based on all these observations, a mechanism for the reaction of Lipo-HT with $\mathrm{Hg}^{2+}$ was proposed as depicted in Figure 6. This involves the rapid formation of the Lipo-HT/ $\mathrm{Hg}^{2+}$ complex, followed by oxidation with formation of a thioketone, likely coupled with the reduction of $\mathrm{Hg}^{2+}$. Hence, 


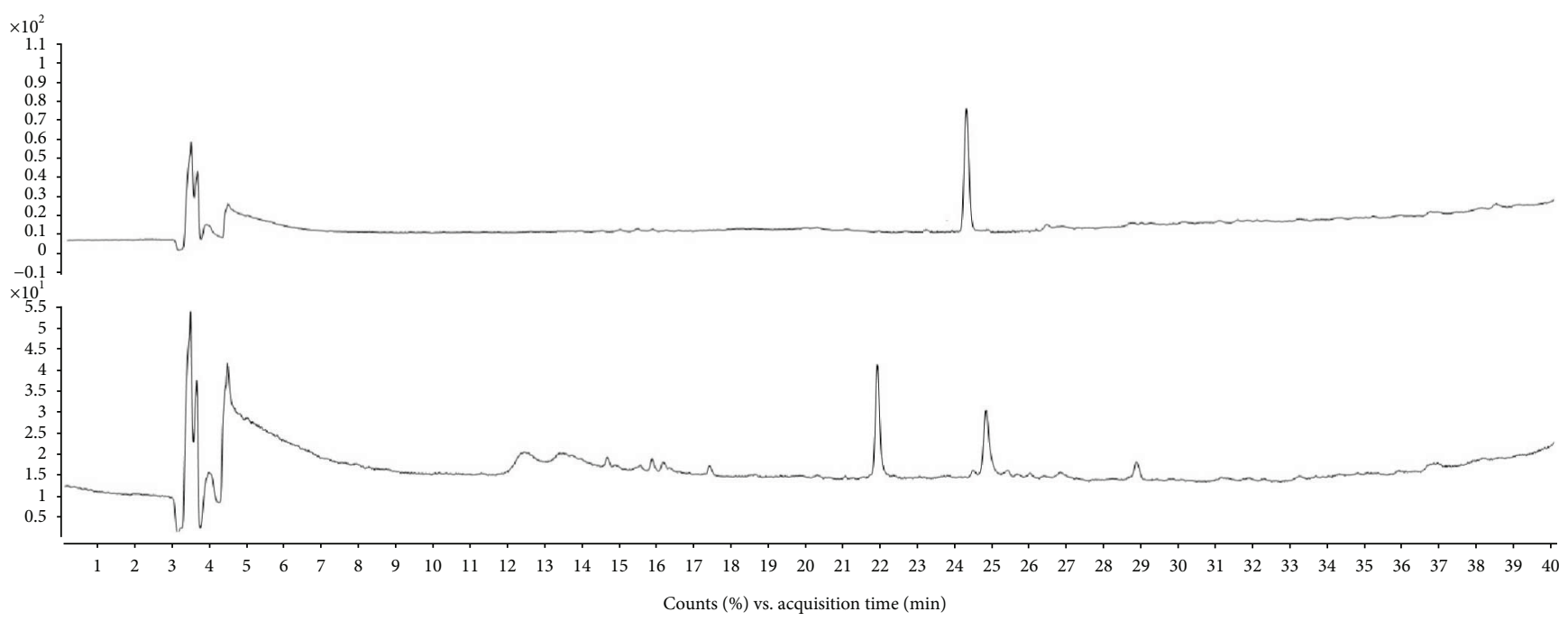

(a)

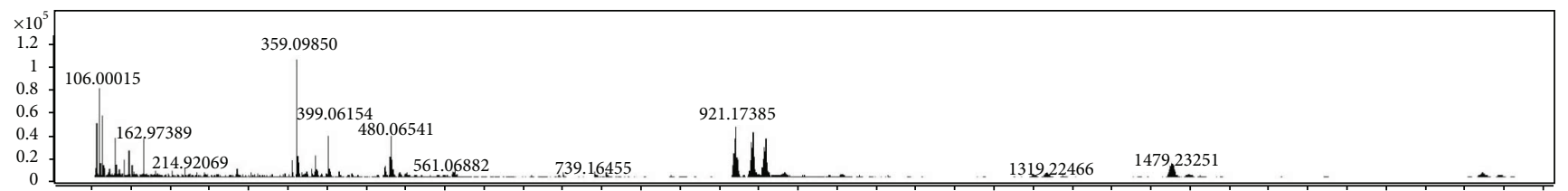

1001502002503003504004505005506006507007508008509009501000105011001150120012501300135014001450150015501600165017001750180018501900

Counts vs. mass to charge $(\mathrm{m} / \mathrm{z})$

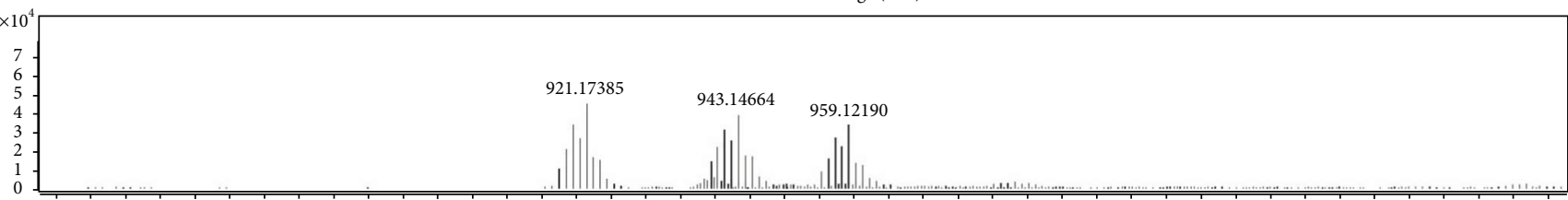

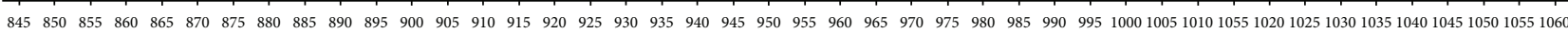
Counts vs. mass to charge $(\mathrm{m} / \mathrm{z})$

(b)

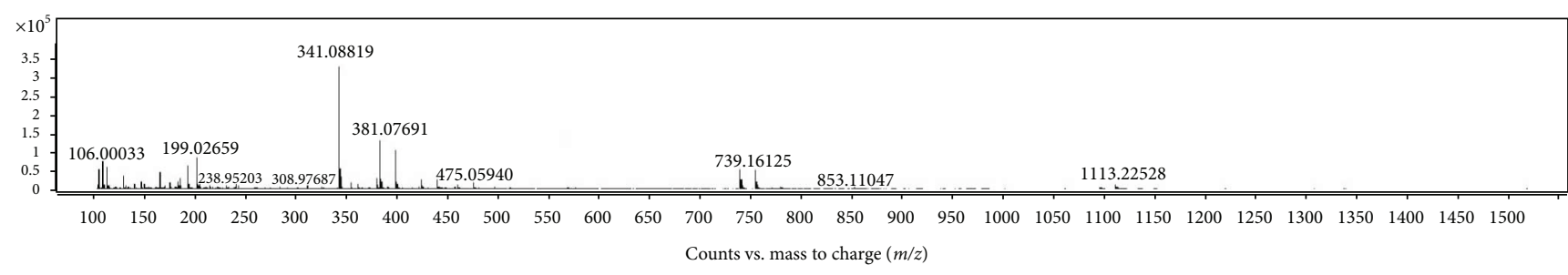

(c)

Figure 5: Analysis of the reaction mixture of Lipo-HT with $\mathrm{Hg}^{2+}$ ions at $\mathrm{pH}$ 7.4. (a) Total ion current (TIC) chromatograms of the reaction mixture of Lipo-HT $(50 \mu \mathrm{M})$ with $\mathrm{Hg}^{2+}(100 \mu \mathrm{M})$ at $\mathrm{pH} 7.4$ (top: before addition of $\mathrm{Hg}^{2+}$, bottom: $5 \mathrm{~min}$ after addition of $\mathrm{Hg}^{2+}$ ). (b) Top: $\mathrm{MS}$ spectrum of the product eluted at $25 \mathrm{~min}$; bottom: inset showing the $\mathrm{Hg}$ isotopic signatures of the complex. (c) MS spectrum of the product eluted at $22 \mathrm{~min}$.

Lipo-HT would act as both a chelating and a reducing agent toward $\mathrm{Hg}$ ions, thus limiting their capacity to induce oxidative damage in biological compartments. Notably, under the same reaction conditions, HT underwent about $30 \%$ consumption after $1 \mathrm{~h}$, giving rise to the methanooxocinobenzodioxinone derivatives identified by comparison of the chromatographic behavior and mass spectra with those of authentic samples [53]. LC/MS analysis of the mixture in the early stages of the reaction revealed the formation of an oxidation product of HT, likely o-quinone; accordingly, the
$\mathrm{UV} / \mathrm{V}$ is spectrum of the reaction mixture at the same time showed an absorption maximum at $390 \mathrm{~nm}$, in close agreement with that reported for the $o$-quinone of HT [49]. No $\mathrm{Hg}$ complex formation could be observed, either by UV/Vis or by LC/MS analysis.

As to the relevance of the results of this model experiment to the effects observed in RBC, it should be noted that both $\mathrm{Hg}^{2+}$ and Lipo-HT and HT concentrations used were higher but comparable to those chosen for the cellular assays. This however does not allow us to extend tout court these 


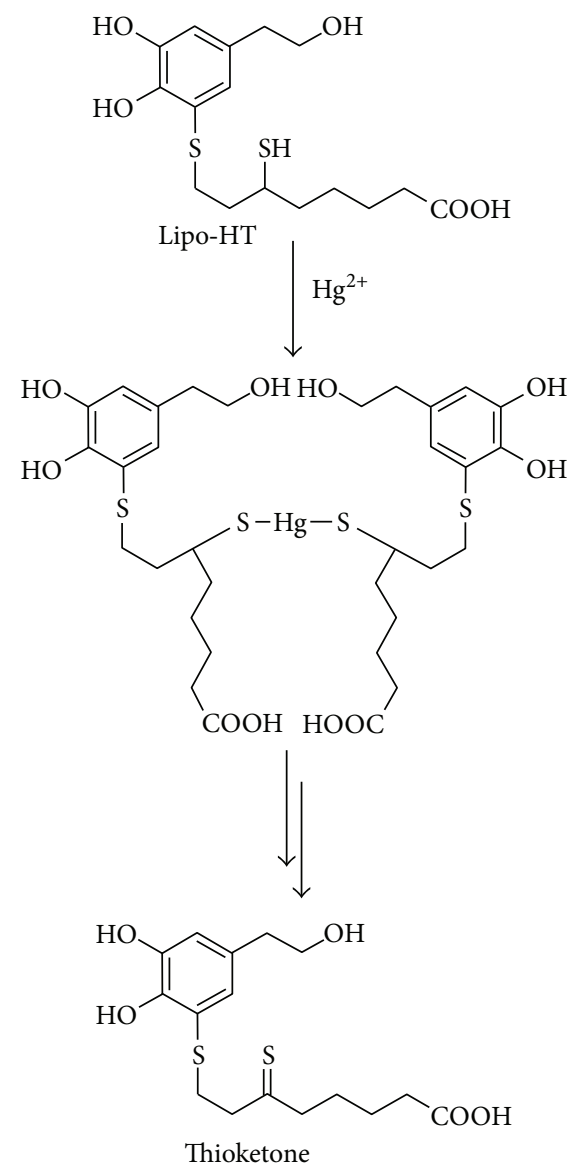

Figure 6: Mechanism proposed for the reaction of Lipo-HT with $\mathrm{Hg}^{2+}$ at $\mathrm{pH}$ 7.4.

findings to the in vivo environment where other species occurring at higher levels may compete with Lipo-HT for Hg binding.

\section{Conclusion}

The use of natural compounds of plant origin with a potential to control the oxidative cellular alterations associated with $\mathrm{Hg}$ exposure is a strategy that is gaining increasing interest to counteract the toxicity of this and other heavy metal pollutants [56]. In this connection, the present investigation provides a significant contribution showing the potency of a derivative of the olive oil polyphenol HT, which contains the active sulfhydryl moiety of dihydrolipoic acid in the molecular scaffold, in controlling the oxidation events triggered by $\mathrm{Hg}$ ions and in protecting the intracellular homeostasis warranted by GSH levels. The effects observed can be ascribed to formation of a $\mathrm{Hg}$ complex involving the free secondary SH group followed by a redox reaction that would spare intracellular GSH. It can therefore be concluded that the greater ability of Lipo-HT to protect RBC from $\mathrm{Hg}$ induced damage compared to HT is likely due to the more effective chelating and reducing capacity toward $\mathrm{Hg}$ ions, in agreement with the higher reducing power toward iron and copper ions previously reported [48]. Although the catechol moiety of Lipo-HT does not seem to be involved in the $\mathrm{Hg}$ complex formation, it may play a role as a scavenger of ROS generated following $\mathrm{Hg}^{2+}$-induced metabolic alterations in the cell. Finally, it should be emphasized that the concentrations of HT and its derivative used in this study (5$20 \mu \mathrm{M}$ ) are in the range of plasma concentrations of HT in individuals who adhere to the Mediterranean dietary habit and consume moderate quantities of extra virgin olive oil $(25 \mathrm{~mL} /$ day). Although further experiments on animal models are clearly needed before the therapeutic use of Lipo-HT against mercury toxicity may be considered, and additional data concerning its absorption, plasma-half-life, and metabolism should be obtained, the results of the present work will expectedly stimulate further studies towards exploitation of this compound in nutraceutical strategies.

\author{
Abbreviations \\ Lipo-HT: 5-S-Lipoylhydroxytyrosol \\ HT: $\quad$ Hydroxytyrosol \\ RBC: $\quad$ Red blood cells \\ ROS: $\quad$ Reactive oxygen species \\ DCFH-DA: $2^{\prime}, 7^{\prime}$-Dichlorodihydrofluorescein diacetate \\ $\mathrm{Hb}$ : Hemoglobin \\ DCF: $\quad$ Dichlorofluorescein \\ DTNB: $\quad 5,5^{\prime}$-Dithiobis(2-nitrobenzoic acid) (Ellman's \\ reagent) \\ TIC: $\quad$ Total ion current \\ MS: $\quad$ Mass spectrometry.
}

\section{Conflicts of Interest}

The authors declare that they have no conflicts of interest.

\section{Authors' Contributions}

The manuscript was written through contributions of all authors. All authors have given approval to the final version of the manuscript.

\section{Acknowledgments}

This work was supported by Research Grants from the University of Campania.

\section{References}

[1] G. del Monaco, A. Officioso, S. D’Angelo et al., "Characterization of extra virgin olive oils produced with typical Italian varieties by their phenolic profile," Food Chemistry, vol. 184, pp. 220-228, 2015.

[2] F. Echeverría, M. Ortiz, R. Valenzuela, and L. Videla, "Hydroxytyrosol and cytoprotection: a projection for clinical interventions," International Journal of Molecular Sciences, vol. 18, no. 12, p. 930, 2017.

[3] H. Poudyal, N. Lemonakis, P. Efentakis et al., "Hydroxytyrosol ameliorates metabolic, cardiovascular and liver changes in a rat model of diet-induced metabolic syndrome: pharmacological and metabolism-based investigation," Pharmacological Research, vol. 117, pp. 32-45, 2017. 
[4] V. Zappia, P. Galletti, C. Manna et al., "Chapter 136 - effects of hydroxytyrosol on cyclosporine nephrotoxicity," in Olives Olive Oil Health Disease Prevention, pp. 1245-1252, Academic Press, San Diego, CA, USA, 2010.

[5] E. Bigagli, L. Cinci, S. Paccosi, A. Parenti, M. D'Ambrosio, and C. Luceri, "Nutritionally relevant concentrations of resveratrol and hydroxytyrosol mitigate oxidative burst of human granulocytes and monocytes and the production of pro-inflammatory mediators in LPS-stimulated RAW 264.7 macrophages," International Immunopharmacology, vol. 43, pp. 147-155, 2017.

[6] S. Bulotta, M. Celano, S. M. Lepore, T. Montalcini, A. Pujia, and D. Russo, "Beneficial effects of the olive oil phenolic components oleuropein and hydroxytyrosol: focus on protection against cardiovascular and metabolic diseases," Journal of Translational Medicine, vol. 12, no. 1, p. 219, 2014.

[7] R. Fabiani, "Anti-cancer properties of olive oil secoiridoid phenols: a systematic review of in vivo studies," Food \& Function, vol. 7, no. 10, pp. 4145-4159, 2016.

[8] C. Manna, L. Tagliafierro, I. Scala, B. Granese, G. Andria, and V. Zappia, "The role of iron toxicity in oxidative stressinduced cellular degeneration in down syndrome: protective effects of phenolic antioxidants," Current Nutrition \& Food Science, vol. 8, no. 3, pp. 206-212, 2012.

[9] A. Napolitano, M. De Lucia, L. Panzella, and M. d'Ischia, "Chapter 134 - The chemistry of tyrosol and hydroxytyrosol: implications for oxidative stress," in Olives and Olive Oil in Health and Disease Prevention, pp. 1225-1232, Academic Press, San Diego, CA, USA, 2010.

[10] H. K. Obied, P. D. Prenzler, I. Konczak, A. U. Rehman, and K. Robards, "Chemistry and bioactivity of olive biophenols in some antioxidant and antiproliferative in vitro bioassays," Chemical Research in Toxicology, vol. 22, no. 1, pp. 227-234, 2009.

[11] A. Napolitano, L. Panzella, M. Savarese et al., "Acid-induced structural modifications of unsaturated fatty acids and phenolic olive oil constituents by nitrite ions: a chemical assessment," Chemical Research in Toxicology, vol. 17, no. 10, pp. 13291337, 2004.

[12] Z. Liu, L. Sun, L. Zhu et al., "Hydroxytyrosol protects retinal pigment epithelial cells from acrolein-induced oxidative stress and mitochondrial dysfunction," Journal of Neurochemistry, vol. 103, no. 6, pp. 2690-2700, 2007.

[13] X. Zhang, J. Cao, L. Jiang, C. Geng, and L. Zhong, "Protective effect of hydroxytyrosol against acrylamide-induced cytotoxicity and DNA damage in HepG2 cells," Mutation Research/ Fundamental and Molecular Mechanisms of Mutagenesis, vol. 664, no. 1-2, pp. 64-68, 2009.

[14] Y. Y. Lee, C. Crauste, H. Wang et al., "Extra virgin olive oil reduced polyunsaturated fatty acid and cholesterol oxidation in rodent liver: is this accounted for hydroxytyrosol-fatty acid conjugation?," Chemical Research in Toxicology, vol. 29, no. 10, pp. 1689-1698, 2016.

[15] C. O. R. Okpala, G. Sardo, S. Vitale, G. Bono, and A. Arukwe, "Hazardous properties and toxicological update of mercury: from fish food to human health safety perspective," Critical Reviews in Food Science and Nutrition, pp. 1-16, 2017.

[16] C. T. Driscoll, R. P. Mason, H. M. Chan, D. J. Jacob, and N. Pirrone, "Mercury as a global pollutant: sources, pathways, and effects," Environmental Science \& Technology, vol. 47, no. 10, pp. 4967-4983, 2013.
[17] K. Sundseth, J. Pacyna, E. Pacyna, N. Pirrone, and R. Thorne, "Global sources and pathways of mercury in the context of human health," International Journal of Environmental Research and Public Health, vol. 14, no. 1, p. 105, 2017.

[18] V. Branco, S. Caito, M. Farina, J. Teixeira da Rocha, M. Aschner, and C. Carvalho, "Biomarkers of mercury toxicity: past, present, and future trends," Journal of Toxicology and Environmental Health, Part B, vol. 20, no. 3, pp. 119154, 2017.

[19] S. Miller, S. Pallan, A. S. Gangji, D. Lukic, and C. M. Clase, "Mercury-associated nephrotic syndrome: a case report and systematic review of the literature," American Journal of Kidney Diseases, vol. 62, no. 1, pp. 135-138, 2013.

[20] A. Carocci, N. Rovito, M. S. Sinicropi, and G. Genchi, "Mercury toxicity and neurodegenerative effects," Reviews of Environmental Contamination and Toxicology, vol. 229, pp. 1-18, 2014.

[21] M. Chin-Chan, J. Navarro-Yepes, and B. Quintanilla-Vega, "Environmental pollutants as risk factors for neurodegenerative disorders: Alzheimer and Parkinson diseases," Frontiers in Cellular Neuroscience, vol. 9, p. 124, 2015.

[22] J. Rooney, "Aplastic anemia, membranous nephropathy and mercury," Indian Journal of Nephrology, vol. 23, no. 6, pp. 467-468, 2013.

[23] G. I. Harisa, A. D. Mariee, O. M. Abo-Salem, and S. M. Attiaa, "Erythrocyte nitric oxide synthase as a surrogate marker for mercury-induced vascular damage: the modulatory effects of naringin," Environmental Toxicology, vol. 29, no. 11, pp. 1314-1322, 2014.

[24] K. Eisele, P. A. Lang, D. S. Kempe et al., "Stimulation of erythrocyte phosphatidylserine exposure by mercury ions," Toxicology and Applied Pharmacology, vol. 210, no. 1-2, pp. 116-122, 2006.

[25] J. K. Virtanen, T. H. Rissanen, S. Voutilainen, and T.-P. Tuomainen, "Mercury as a risk factor for cardiovascular diseases," The Journal of Nutritional Biochemistry, vol. 18, no. 2, pp. 75-85, 2007.

[26] S. Omanwar and M. Fahim, "Mercury exposure and endothelial dysfunction," International Journal of Toxicology, vol. 34, no. 4, pp. 300-307, 2015.

[27] Z. Chen, X. Wu, H. Luo et al., "Acute exposure of mercury chloride stimulates the tissue regeneration program and reactive oxygen species production in the Drosophila midgut," Environmental Toxicology and Pharmacology, vol. 41, pp. 32-38, 2016.

[28] H. Ghizoni, V. de Souza, M. R. Straliotto, A. F. de Bem, M. Farina, and M. A. Hort, "Superoxide anion generation and oxidative stress in methylmercury-induced endothelial toxicity in vitro," Toxicology In Vitro, vol. 38, pp. 19-26, 2017.

[29] J. P. K. Rooney, "The role of thiols, dithiols, nutritional factors and interacting ligands in the toxicology of mercury," Toxicology, vol. 234, no. 3, pp. 145-156, 2007.

[30] P. D. Oram, X. Fang, Q. Fernando, P. Letkeman, and D. Letkeman, "The formation constants of mercury(II)-glutathione complexes," Chemical Research in Toxicology, vol. 9, no. 4, pp. 709-712, 1996.

[31] L. E. Hernández, J. Sobrino-Plata, M. B. Montero-Palmero et al., "Contribution of glutathione to the control of cellular redox homeostasis under toxic metal and metalloid stress," Journal of Experimental Botany, vol. 66, no. 10, pp. 29012911, 2015. 
[32] Y. Elseady and E. Zahran, "Ameliorating effect of $\beta$-carotene on antioxidant response and hematological parameters of mercuric chloride toxicity in Nile tilapia (Oreochromis niloticus)," Fish Physiology and Biochemistry, vol. 39, no. 4, pp. 1031-1041, 2013.

[33] Y. Deng, Z. Xu, W. Liu, H. Yang, B. Xu, and Y. Wei, "Effects of lycopene and proanthocyanidins on hepatotoxicity induced by mercuric chloride in rats," Biological Trace Element Research, vol. 146, no. 2, pp. 213-223, 2012.

[34] W. R. García-Niño and J. Pedraza-Chaverrí, "Protective effect of curcumin against heavy metals-induced liver damage," Food and Chemical Toxicology, vol. 69, pp. 182-201, 2014.

[35] Y. J. Shin, J. J. Kim, Y. J. Kim et al., "Protective effects of quercetin against $\mathrm{HgCl}_{2}$-induced nephrotoxicity in SpragueDawley rats," Journal of Medicinal Food, vol. 18, no. 5, pp. 524-534, 2015.

[36] J. L. Franco, H. C. Braga, J. Stringari et al., "Mercurial-induced hydrogen peroxide generation in mouse brain mitochondria: protective effects of quercetin," Chemical Research in Toxicology, vol. 20, no. 12, pp. 1919-1926, 2007.

[37] L. Tagliafierro, A. Officioso, S. Sorbo, A. Basile, and C. Manna, "The protective role of olive oil hydroxytyrosol against oxidative alterations induced by mercury in human erythrocytes," Food and Chemical Toxicology, vol. 82, pp. 59-63, 2015.

[38] A. Officioso, K. Alzoubi, F. Lang, and C. Manna, "Hydroxytyrosol inhibits phosphatidylserine exposure and suicidal death induced by mercury in human erythrocytes: possible involvement of the glutathione pathway," Food and Chemical Toxicology, vol. 89, pp. 47-53, 2016.

[39] K. M. Lim, S. Kim, J. Y. Noh et al., "Low-level mercury can enhance procoagulant activity of erythrocytes: a new contributing factor for mercury-related thrombotic disease," Environmental Health Perspectives, vol. 118, no. 7, pp. 928-935, 2010.

[40] V. Mohan, S. Das, and S. B. S. Rao, "Hydroxytyrosol, a dietary phenolic compound forestalls the toxic effects of methylmercury-induced toxicity in IMR-32 human neuroblastoma cells," Environmental Toxicology, vol. 31, no. 10, pp. 1264-1275, 2016.

[41] C. Manna, V. Migliardi, F. Sannino, A. De Martino, and R. Capasso, "Protective effects of synthetic hydroxytyrosol acetyl derivatives against oxidative stress in human cells," Journal of Agricultural and Food Chemistry, vol. 53, no. 24, pp. 96029607, 2005.

[42] M. V. Sepporta, M. Á. López-García, R. Fabiani, I. Maya, and J. G. Fernández-Bolaños, "Enhanced chemopreventive activity of hydroxytyrosol on HL60 and HL60R cells by chemical conversion into thio derivatives," European Journal of Pharmaceutical Sciences, vol. 48, no. 4-5, pp. 790-798, 2013.

[43] R. Bernini, M. S. Gilardini Montani, N. Merendino, A. Romani, and F. Velotti, "Hydroxytyrosol-derived compounds: a basis for the creation of new pharmacological agents for cancer prevention and therapy," Journal of Medicinal Chemistry, vol. 58, no. 23, pp. 9089-9107, 2015.

[44] I. Fernandez-Pastor, A. Fernandez-Hernandez, F. Rivas, A. Martinez, A. Garcia-Granados, and A. Parra, "Synthesis and antioxidant activity of hydroxytyrosol alkyl-carbonate derivatives," Journal of Natural Products, vol. 79, no. 7, pp. 1737-1745, 2016.

[45] M. H. Gordon, F. Paiva-Martins, and M. Almeida, "Antioxidant activity of hydroxytyrosol acetate compared with that of other olive oil polyphenols," Journal of Agricultural and Food Chemistry, vol. 49, no. 5, pp. 2480-2485, 2001.
[46] I. Medina, S. Lois, D. Alcantara, R. Lucas, and J. C. Morales, "Effect of lipophilization of hydroxytyrosol on its antioxidant activity in fish oils and fish oil-in-water emulsions," Journal of Agricultural and Food Chemistry, vol. 57, no. 20, pp. 9773-9779, 2009.

[47] R. Amorati, L. Valgimigli, L. Panzella, A. Napolitano, and M. d'Ischia, "5-S-lipoylhydroxytyrosol, a multidefense antioxidant featuring a solvent-tunable peroxyl radical-scavenging 3-thio-1,2-dihydroxybenzene motif," Journal of Organic Chemistry, vol. 78, no. 19, pp. 9857-9864, 2013.

[48] L. Panzella, L. Verotta, L. Goya et al., "Synthesis and bioactivity profile of 5-S-lipoylhydroxytyrosol-based multidefense antioxidants with a sizeable (poly)sulfide chain," Journal of Agricultural and Food Chemistry, vol. 61, no. 8, pp. 1710-1717, 2013.

[49] M. De Lucia, L. Panzella, A. Pezzella, A. Napolitano, and M. d'Ischia, "Plant catechols and their S-glutathionyl conjugates as antinitrosating agents: expedient synthesis and remarkable potency of 5-S-glutathionylpiceatannol," Chemical Research in Toxicology, vol. 21, no. 12, pp. 2407-2413, 2008.

[50] M. d'Ischia, A. Napolitano, P. Manini, and L. Panzella, "Secondary targets of nitrite-derived reactive nitrogen species: nitrosation/nitration pathways, antioxidant defense mechanisms and toxicological implications," Chemical Research in Toxicology, vol. 24, no. 12, pp. 2071-2092, 2011.

[51] D. Yang, X. Tan, Z. Lv et al., "Regulation of Sirt1/Nrf2/TNF- $\alpha$ signaling pathway by luteolin is critical to attenuate acute mercuric chloride exposure induced hepatotoxicity," Scientific Reports, vol. 6, no. 1, article 37157, 2016.

[52] J. J. M. van den Berg, J. A. F. op den Kamp, B. H. Lubin, B. Roelofsen, and F. A. Kuypers, "Kinetics and site specificity of hydroperoxide-induced oxidative damage in red blood cells," Free Radical Biology \& Medicine, vol. 12, no. 6, pp. 487-498, 1992.

[53] D. Vogna, A. Pezzella, L. Panzella, A. Napolitano, and M. d'Ischia, "Oxidative chemistry of hydroxytyrosol: isolation and characterisation of novel methanooxocinobenzodioxinone derivatives," Tetrahedron Letters, vol. 44, no. 45, pp. 8289-8292, 2003.

[54] X. Lin, J. Brooks, M. Bronson, and M. Ngu-Schwemlein, "Evaluation of the association of mercury(II) with some dicysteinyl tripeptides," Bioorganic Chemistry, vol. 44, pp. 8-18, 2012.

[55] M. Farid, H. Khan, H. Khan, R. Z. Paracha, and G. M. Khan, "Effect of mercuric chloride on glutathione (GSH) level in plasma and cytosolic fraction of human blood," Jordan Journal of Pharmaceutical Science, vol. 2, no. 1, pp. 22-31, 2008.

[56] P. Manini, L. Panzella, T. Eidenberger et al., "Efficient binding of heavy metals by black sesame pigment: toward innovative dietary strategies to prevent bioaccumulation," Journal of Agricultural and Food Chemistry, vol. 64, no. 4, pp. 890-897, 2016. 


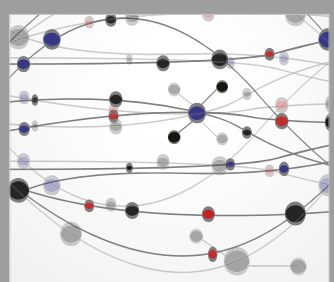

The Scientific World Journal
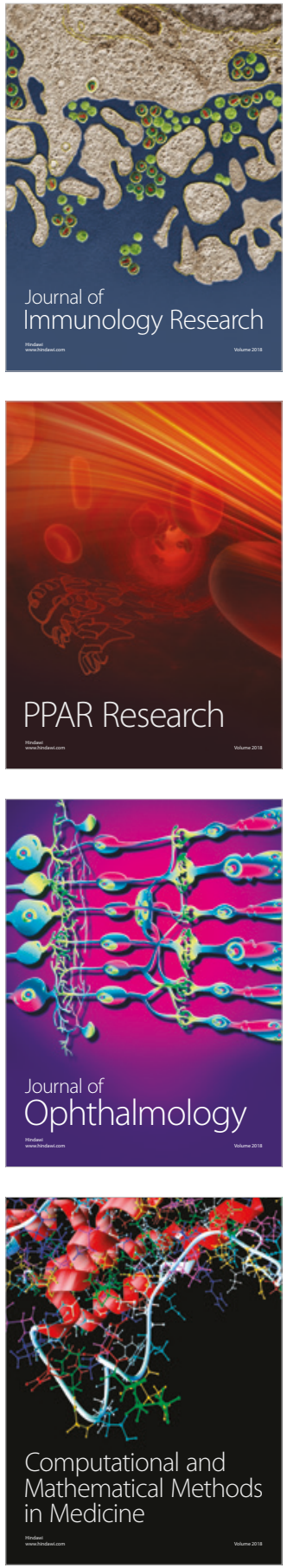

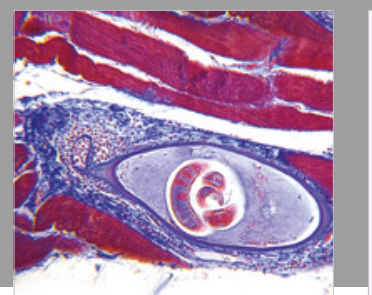

Gastroenterology Research and Practice

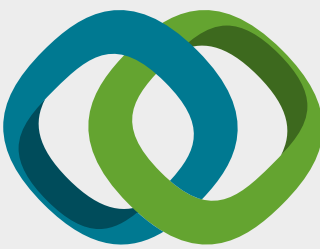

\section{Hindawi}

Submit your manuscripts at

www.hindawi.com
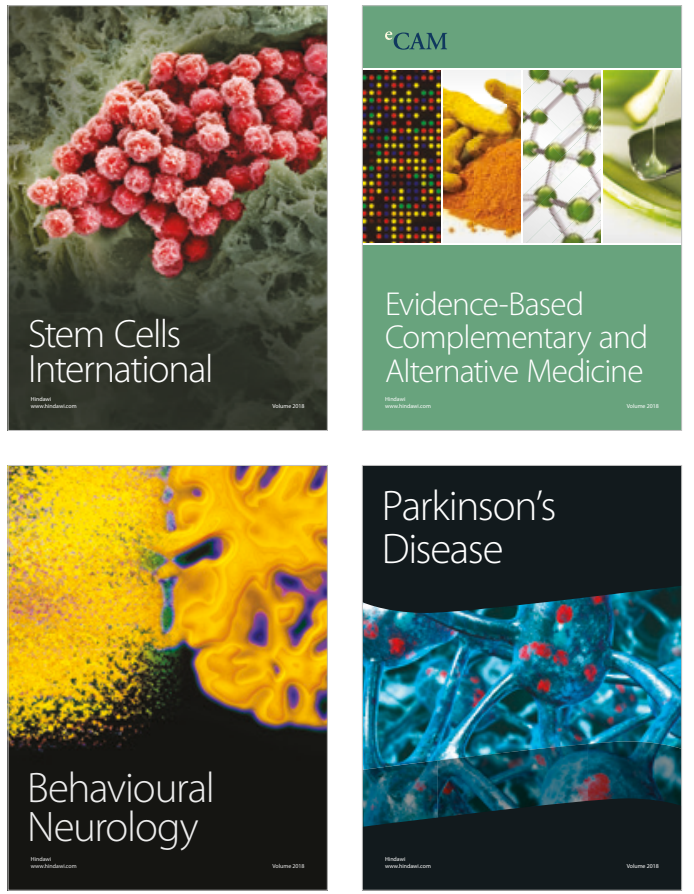

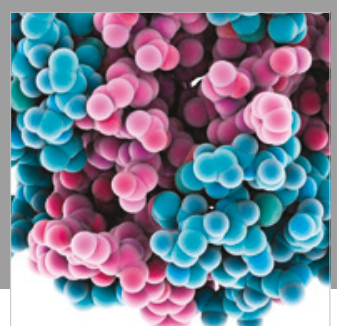

ournal of

Diabetes Research

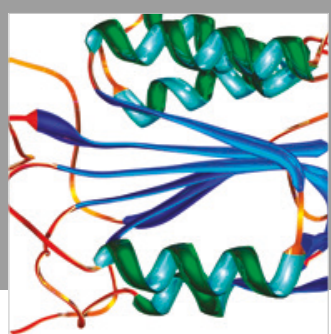

Disease Markers
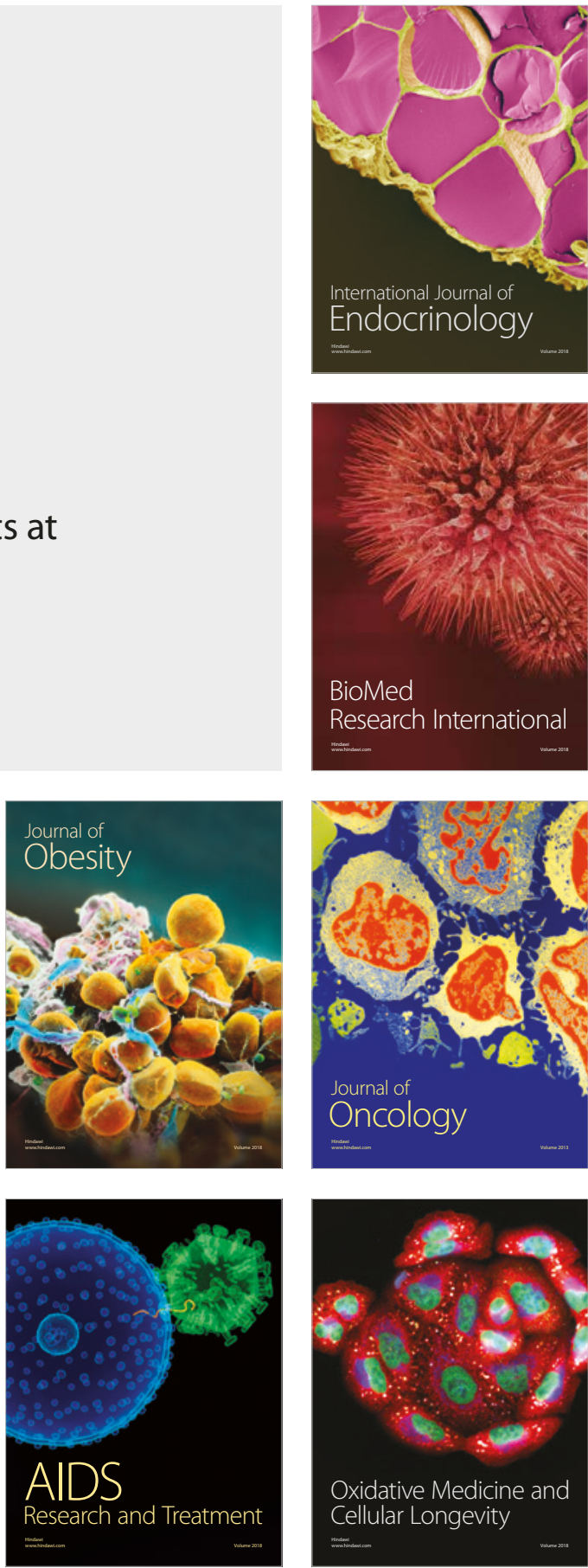